\title{
Insecticide resistance and role in malaria transmission of Anopheles funestus populations from Zambia and Zimbabwe
}

Kwang S Choi ${ }^{1,2,3}$, Riann Christian ${ }^{1,2}$, Luisa Nardini ${ }^{1,2}$, Oliver R Wood ${ }^{1,2}$, Eunice Agubuzo ${ }^{1,2}$, Mbanga Muleba $^{4}$, Shungu Munyati ${ }^{5}$, Aramu Makuwaza ${ }^{6}$, Lizette L Koekemoer ${ }^{1,2}$, Basil D Brooke ${ }^{1,2}$, Richard H Hunt ${ }^{1}$ and Maureen Coetzee ${ }^{1,2^{*}}$

\begin{abstract}
Background: Two mitochondrial DNA clades have been described in Anopheles funestus populations from southern Africa. Clade I is common across the continent while clade II is known only from Mozambique and Madagascar. The specific biological status of these clades is at present unknown. We investigated the possible role that each clade might play in the transmission of Plasmodium falciparum and the insecticide resistance status of An. funestus from Zimbabwe and Zambia.

Methods: Mosquitoes were collected inside houses from Nchelenge District, Zambia and Honde Valley, Zimbabwe in 2013 and 2014. WHO susceptibility tests, synergist assays and resistance intensity tests were conducted on wild females and progeny of wild females. ELISA was used to detect Plasmodium falciparum circumsporozoite protein. Specimens were identified to species and mtDNA clades using standard molecular methods.

Results: The Zimbabwean samples were all clade I while the Zambian population comprised $80 \%$ clade I and 20\% clade II in both years of collection. ELISA tests gave an overall infection rate of $2.3 \%$ and $2.1 \%$ in 2013 , and $3.5 \%$ and 9.2\% in 2014 for Zimbabwe and Zambia respectively. No significant difference was observed between the clades. All populations were resistant to pyrethroids and carbamates but susceptible to organochlorines and organophosphates. Synergist assays indicated that pyrethroid resistance is mediated by cytochrome P450 mono-oxygenases. Resistance intensity tests showed high survival rates after 8-hrs continuous exposure to pyrethroids but exposure to bendiocarb gave the same results as the susceptible control.

Conclusions: This is the first record of An. funestus mtDNA clade II occurring in Zambia. No evidence was found to suggest that the clades are markers of biologically separate populations. The ability of An. funestus to withstand prolonged exposure to pyrethroids has serious implications for the use of these insecticides, either through LLINs or IRS, in southern Africa in general and resistance management strategies should be urgently implemented.
\end{abstract}

Keywords: Anopheles funestus, mtDNA clades, Insecticide resistance, Resistance intensity, Plasmodium infection

\footnotetext{
* Correspondence: maureenc@nicd.ac.za

${ }^{1}$ Wits Research Institute for Malaria, School of Pathology, Faculty of Health

Sciences, University of the Witwatersrand, Johannesburg, South Africa

${ }^{2}$ Centre for Opportunistic, Tropical and Hospital Infections, National Institute

for Communicable Diseases, National Health Laboratory Service,

Johannesburg, South Africa

Full list of author information is available at the end of the article
} 


\section{Background}

In the recent past, malaria vector control has been primarily pyrethroid-based through indoor residual house spraying [IRS] or the use of long-lasting insecticide-treated bed nets (LLINs) or both. This intensive use of insecticides, together with pesticide usage in agriculture, has led to a dramatic increase in resistance in the mosquito vectors across the whole African continent [http://www.irmapper.com]. Anopheles funestus, one of the four major vectors of Plasmodium falciparum malaria in Africa [1,2], is widely distributed throughout much of the African tropics and subtropics [3]. The earliest records of insecticide resistance in An. funestus are from the 1980s [4] and currently resistance in this species is known from Mali, Guinea, Ghana, Benin, Uganda, Kenya, Malawi, Zambia, Mozambique and South Africa [1]. A well-documented impact of insecticide resistance on vector control programme failure is the major malaria epidemic that occurred in South Africa in 1999/2000 when pyrethroid resistant An. funestus returned to South Africa after DDT was replaced with pyrethroids for indoor house spraying [5]. The situation returned to pre-failure levels after DDT was reintroduced and used in a mosaic system together with pyrethroids. A number of insecticide resistance studies on An. funestus from southern Africa have been documented, but studies from other regions are limited [1].

Anopheles funestus belongs to a group of at least 11 species, all of which are morphologically similar at the adult stage [6,7]. Some members of the group can be distinguished on egg and larval characteristics (An. confusus, $A n$. rivulorum and $A n$. leesoni) but this is not always the case and those species belonging to the An. funestus subgroup (An. funestus, An. funestus-like, An. parensis, An. aruni and $A n$. vaneedeni) are virtually identical [1,6,7]. The importance of species identification lies in the fact that of the 11 species, only An. funestus plays a major role in malaria transmission, this species being highly adapted to humans and human habitation with parasite infection rates sometimes as high as $22 \%$ [6]. The only other member of the group that has been implicated in low-level, localised transmission is An. rivulorum in Tanzania [8] and western Kenya [9] but in each case the parasite infection rates have been below $1 \%$.

Malaria vector control programmes need to know that their interventions are targeting the major vectors so that scarce resources are not wasted on non-vector mosquitoes. As a result, various genetical techniques have been applied to members of the An. funestus group with the aim of providing accurate species identification. Initially, the banding patterns of the giant polytene chromosomes were used $[10,11]$ but these have limited practical value because $A n$. funestus and $A n$. vaneedeni share the same inversion arrangements resulting in the same banding patterns [10], as do An. funestus and An. funestus-like
[12]. In addition, the technique has the disadvantage of being applicable only to half-gravid female mosquitoes. However, studies of the chromosomal inversion polymorphisms in Burkina Faso revealed the existence of two distinct forms that apparently do not mate in sympatry [13] and that are also significantly differentiated at the molecular level [14], supporting the hypothesis of two distinct species being present. Twenty years after the work of Green and Hunt [10,11], a molecular assay was developed that allowed for the rapid and accurate identification of the five most common members of the An. funestus group [15] and this is the standard protocol used in many laboratories today.

While An. funestus has abundant levels of molecular and chromosomal polymorphism across its range, there are only a few population genetic studies of the species and these are limited in geographic scope compared with similar work on the Anopheles gambiae complex [1]. Phylogenetic studies using mitochondrial DNA (mtDNA) have revealed that there are two clades within An. funestus. Clade I is widespread throughout the continent and clade II is known only from southern Mozambique and Madagascar [16,17]. It is estimated that these two clades evolved independently about 1 million years ago based on fixed differences and divergence in mtDNA [16]. However, the deep mtDNA divergence was not accompanied by corresponding nuclear divergence as measured by 10 microsatellite loci in the same study [16] so it is not clear what this genetic variation means in terms of species differentiation.

In the present study, we investigated the biological attributes of insecticide resistance and parasite infection rates that both impact on malaria vector control activities, in relation to the mtDNA clades in Zambia and Zimbabwe.

\section{Methods}

\section{Field collections}

Anopheles funestus specimens were collected resting inside houses from Honde Valley, Zimbabwe [ $18^{\circ} 23.161^{\prime} \mathrm{S}$, $32^{\circ} 59.946^{\prime} \mathrm{E}$ ] and Nchelenge District, Zambia [9 $9^{\circ} 19.115^{\prime} \mathrm{S}$, $\left.28^{\circ} 45.070^{\prime} \mathrm{E}\right]$. Permission to enter houses was obtained from the village headmen and from each individual house owner. Collections were carried out between February and April 2013. In February and March 2014, the most productive houses identified from the 2013 survey, were visited with the intention of maximizing the sample size over the few days that were available for collecting.

\section{Insecticide susceptibility tests}

The WHO insecticide resistance tests [18] were performed in the field (Zambia both years; Zimbabwe 2013 only, 2014 tests were carried out on F-1 progeny) to investigate the susceptibility status of wild-caught $A n$. 
funestus females to WHO diagnostic doses of pyrethroids (0.05\% deltamethrin and $0.05 \%$ lambda-cyhalothrin), carbamates $(0.1 \%$ bendiocarb and $0.1 \%$ propoxur), organochlorines (4.0\% DDT and 4.0\% dieldrin) and organophosphates (5.0\% malathion, $1.0 \%$ fenitrothion and $0.25 \%$ pirimiphosmethyl). The age of the mosquitoes used for the tests was unknown and the physiological status ranged from blood fed to gravid. Each exposure consisted of $\sim 25$ randomly selected mosquitoes for the seven insecticides plus controls. Exposure time for six of the insecticides was 1 hour while mosquitoes were exposed to fenitrothion for 2 hours [18]. The total number of mosquitoes exposed to each insecticide was dependent on the numbers of mosquitoes available in the field and the initial results obtained for each insecticide. Final mortality was recorded 24 hours postexposure. Efficacy of WHO insecticide papers was confirmed before and after the field tests using a known susceptible mosquito strain (FANG - An. funestus originating from Angola and colonised in 2003). All exposed specimens were stored on silica gel and returned to the laboratory for further processing.

A proportion of the collection from both localities was brought back to the laboratory alive. In the insectary, the wild females were set up for egg-laying in small glass vials lined with oval pieces of filter paper with a small amount of water in the bottom [9,19] (Figure 1) and held in wooden racks. This method usually results in $>70 \%$ of wild females laying eggs. The Zimbabwe collections yielded 92 egg batches in 2013 and 140 in 2014. The Zambia collections yielded 148 clade I and 40 clade II egg batches in 2013, and 265 clade I and 67 clade II in 2014. Egg batches were reared individually until species/ clade identification was obtained, at which point they were pooled into their separate groups. First generation adults, aged 2-5 days, were used for synergist and resistance intensity assays and, in the case of the 2014 Zimbabwe collections, also for WHO susceptibility tests.

\section{Resistance intensity assays}

Female Zimbabwe and Zambia samples were exposed to 0.05\% deltamethrin, $0.1 \%$ bendiocarb and 0.05\% lambdacyhalothrin (Zimbabwe samples only) treated papers continuously for 8 hours with knockdown being recorded at various time intervals. The WHO test tubes were laid flat so that once knocked down, the mosquitoes continued to be exposed to the insecticide. The 8-hr cut-off was arbitrarily chosen as the likely time a mosquito might come into contact with a sprayed wall after taking a blood meal. The susceptible laboratory strain FANG was used as a control.

\section{Synergist bioassays}

Synergist assays [20] were performed by exposing 153 clade I specimens from Zimbabwe, 128 clade I and 113 clade II specimens from Zambia to $4 \%$ piperonyl butoxide (PBO), an inhibitor of monooxygenases, for $1 \mathrm{~h}$ prior to exposing them to deltamethrin and bendiocarb. Controls consisted of specimens exposed to the insecticides alone, and to $\mathrm{PBO}$ alone. The samples for the synergist assays were $2-3$ day old unfed, $\mathrm{F}_{1}$ females.

\section{Laboratory analyses}

Specimens were initially identified morphologically in the field using the keys of Gillies and Coetzee [7]. DNA was extracted from either legs or wings of individual mosquitoes using a prepGEM insect DNA extraction kit (ZyGEM, New Zealand) following the manufacturer's instructions. Briefly, legs or wings of mosquitoes were

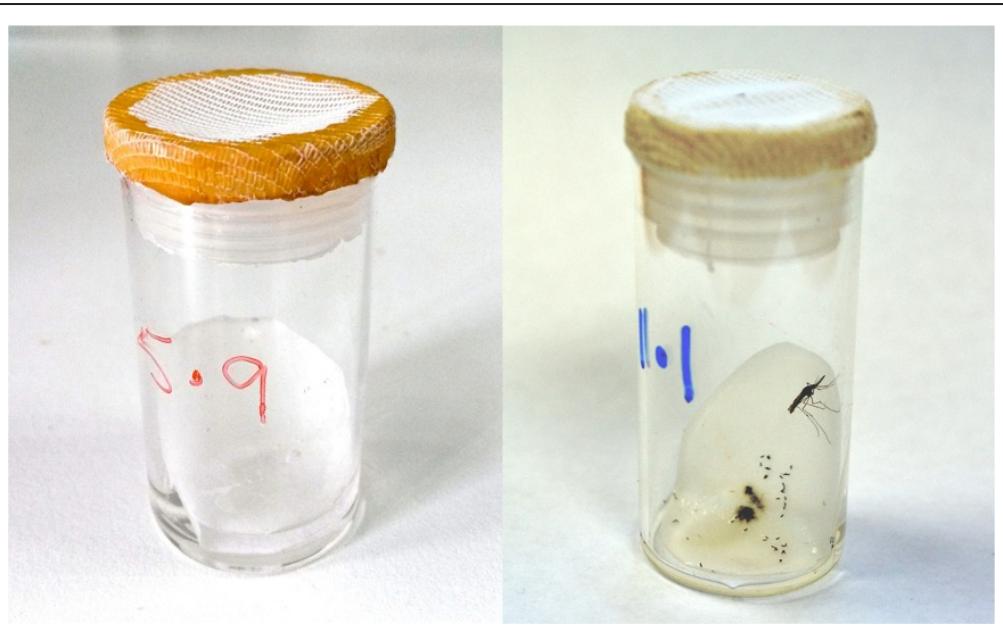

Figure 1 A very simple method for inducing egg-laying in wild Anopheles funestus that consistently results in $>70 \%$ of the females ovipositing. Glass vials, $45 \mathrm{~mm}$ high $\times 25 \mathrm{~mm}$ diameter, with gauze lids are used. Small pieces of filter paper are placed at an angle in the bottom of the tube with approximately $1 \mathrm{ml}$ water for egg laying. Females, resting inside the plastic lids, can be transferred to clean vials once they have laid eggs, facilitating blood-feeding for multiple egg batches. 
Table 1 Identification of clades and P. falciparum infection rates by ELISA for An. funestus from Zimbabwe and Zambia

\begin{tabular}{|c|c|c|c|c|c|c|}
\hline & \multirow[t]{2}{*}{$n$} & \multicolumn{2}{|l|}{ Clades } & \multicolumn{3}{|l|}{ ELISA } \\
\hline & & $I$ & II & Clade I & Clade II & Total \\
\hline Zimbabwe 2013 & 342 & $92(100 \%)$ & - & 7/303 (2.3\%) & - & $7 / 303(2.3 \%)$ \\
\hline Zimbabwe 2014 & 140 & 88 (100\%) & - & 4/115 (3.5\%) & - & 4/115 (3.5\%) \\
\hline Zambia 2013 & 513 & $413(80.5 \%)$ & $100(19.5 \%)$ & $8 / 346$ (2.3\%) & $1 / 76(1.3 \%)$ & 9/422 (2.1\%) \\
\hline Zambia 2014 & 332 & 264 (79.5\%) & 68 (20.5\%) & 17/171 (9.9\%) & $5 / 68$ (7.4\%) & $22 / 239(9.2 \%)$ \\
\hline
\end{tabular}

homogenized with reagents, incubated for 15 minutes at $75^{\circ} \mathrm{C}$ and then for 5 minutes at $95^{\circ} \mathrm{C}$. A total of $10 \mu \mathrm{L}$ of solution from each sample was produced from the extraction procedure. All specimens were identified to species using the method of Koekemoer et al. [15]. After PCR species identification, a subsample of An. funestus was identified to clades using the hydrolysis probe analysis (Taqman assay) [21].

Plasmodium falciparum parasite infection in mosquitoes was detected from heads and thoraces of individual females using the enzyme-linked immunosorbent assay (ELISA) [22]. To validate the results, all of the positive samples were re-analyzed with the ELISA after heating the ELISA-homogenates in a heat block for 10 minutes at $100^{\circ} \mathrm{C}$ [23]. A positive control (recombinant P. falciparum) and seven negative controls (uninfected insectary female $A n$. funestus) were included. An Ascent Multiskan (RC vl. 5.0, Genesis version 3.03, Labsystems) platereader was used for the analysis of data. The number of specimens that remained positive after heating the lysate was used to calculate the sporozoite rate.

\section{Data analysis}

Resistance intensity assay data were compared using oneway ANOVA (Statistix 8 -Talahasee, Fl, USA). Variations in mortality between synergised and unsynergised samples were assessed using Chi-square (Statistix 8 -Talahasee, Fl, USA).

\section{Results}

Identification of An. funestus

All specimens were identified morphologically as belonging to the An. funestus group [7]. Table 1 presents the results of molecular species confirmation and clade identifications by year [15,21] of a total of 482 and 845 specimens of $A n$. funestus from Zimbabwe and Zambia respectively. The Taqman assay used for mtDNA clade identification [21] revealed that only clade I was present in Zimbabwe, although sample sizes were relatively small and clade II might have been missed. In Zambia, 677 specimens were identified as clade I (80\%) and 168 as clade II (20\%). These proportions did not differ between 2013 and 2014 collections. A single specimen of An. leesoni was identified by PCR.

\section{Enzyme-linked immunosorbent assays (ELISA)}

The Plasmodium falciparum infection rates are given in Table 1. A total of 725 female mosquitoes were tested using the original ELISA method [22] with a second reanalysis of positive specimens using the heated ELISA lysate method [23] to confirm the results. In 2013, the infection rates were $2.3 \%$ for clade I from both Zimbabwe

Table 2 Insecticide susceptibility tests of total An. funestus from Zimbabwe and Zambia for two consecutive years

\begin{tabular}{|c|c|c|c|c|}
\hline & \multicolumn{2}{|l|}{ Zimbabwe } & \multicolumn{2}{|l|}{ Zambia } \\
\hline & \%24 hr mortality (n) 2013 & $\% 24$ hr mortality (n) 2014 & $\% 24$ hr mortality (n) 2013 & $\% 24$ hr mortality (n) 2014 \\
\hline Deltamethrin & $65.3(49)$ & $85.1(94)$ & $47.2(72)$ & $45.5(99)$ \\
\hline Lambda-cyhalothrin & $24.1(83)$ & $3.7(54)$ & $19.0(79)$ & - \\
\hline Bendiocarb & $70.1(67)$ & $73.1(104)$ & $75.5(102)$ & $45.2(104)$ \\
\hline Propoxur & $77.4(53)$ & - & $60.5(76)$ & $79.3(92)$ \\
\hline DDT & $100(55)$ & $100(123)$ & $100(73)$ & $100(62)$ \\
\hline Dieldrin & $100(43)$ & - & $100(68)$ & $100(56)$ \\
\hline Malathion & $100(48)$ & - & $100(106)$ & $99(95)$ \\
\hline Fenitrothion & $100(56)$ & - & $100(92)$ & $100(43)$ \\
\hline Pirimiphos-methyl & - & $100(104)$ & - & $100(96)$ \\
\hline Controls & $0(82)$ & $1.9(104)$ & $0(94)$ & $4.8(83)$ \\
\hline
\end{tabular}


Table 3 Status of insecticide resistance for the clades of wild An. funestus females from Zambia in 2013

\begin{tabular}{lllll}
\hline & Clade I & \multicolumn{3}{c}{ Clade II } \\
\cline { 2 - 5 } & $\begin{array}{l}\text { Sample } \\
\text { size }\end{array}$ & $\begin{array}{l}\text { \% 24 hr } \\
\text { mortality }\end{array}$ & $\begin{array}{l}\text { Sample } \\
\text { size }\end{array}$ & $\begin{array}{l}\text { \% 24 hr } \\
\text { mortality }\end{array}$ \\
\hline Deltamethrin & 59 & 42.4 & 13 & 76.9 \\
Lambda-cyhalothrin & 60 & 18.3 & 19 & 21.0 \\
Bendiocarb & 83 & 77.5 & 19 & 73.7 \\
Propoxur & 67 & 57.6 & 9 & 77.3 \\
\hline
\end{tabular}

and Zambia while the Zambian clade II samples had an infection rate of $1.3 \%$. In 2014, both localities showed an increase in sporozoite rates with 3.5\% in Zimbabwe, 9.9\% in Zambia clade I and $7.4 \%$ in clade II.

\section{Insecticide resistance WHO bioassays}

Insecticide resistance tests were carried out using the standard WHO methods and diagnostic doses [18] for two pyrethroids, two carbamates, two organochlorines and three organophosphates. Anopheles funestus from Zimbabwe and Zambia were all susceptible to the organochlorines and the organophosphates but resistant to pyrethroids and carbamates (Table 2). Table 3 gives the susceptibility results for the two clades in Zambia to pyrethroids and carbamates but given that the sample sizes are very small, these results require further investigation. In all cases, $A n$. funestus meets the WHO criteria for resistance (i.e. $<90 \%$ mortality) to both pyrethroids and carbamates [18].

\section{Resistance intensity experiments}

The results are shown in Figures 2, 3, 4, 5, 6, 7 and Table 4. Data points in Figures 2, 3, 4, 5, 6 and 7 are overall percentage knock-downs across all replicates. Both Zimbabwe clade I and Zambian clades I and II showed similar results for 8-hr exposures to deltamethrin that were significantly different to the FANG

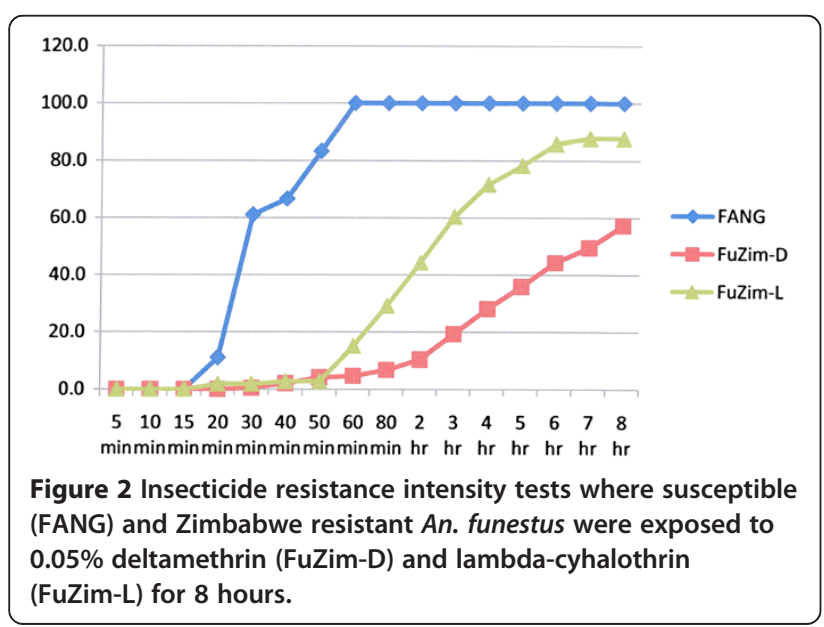

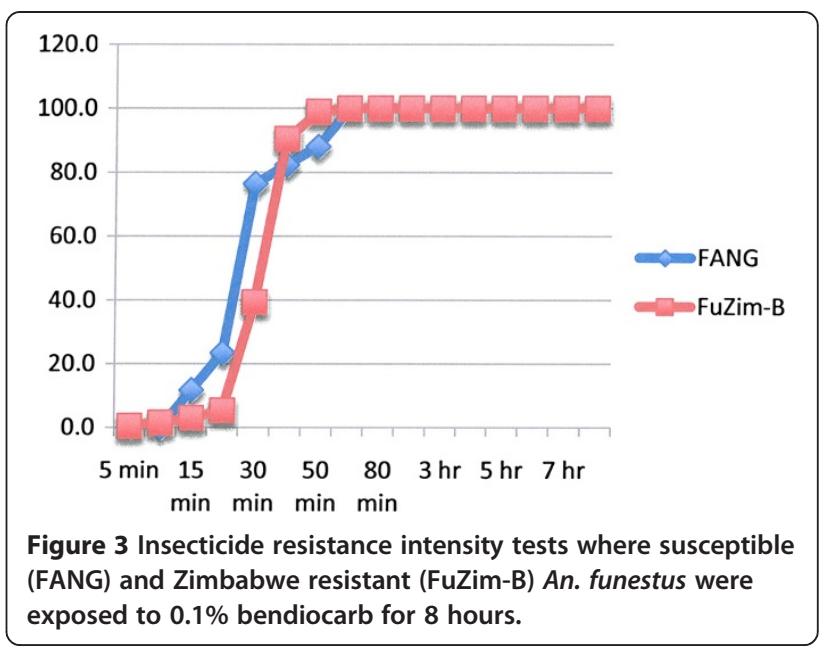

results (ANOVA: $\mathrm{df}=1 ; \mathrm{P}<0.01$ in all cases) (Figures 2, 4 and 5). There were sufficient numbers of Zimbabwe $\mathrm{F}_{1} \mathrm{~s}$ to carry out tests on lambda-cyhalothrin and the results showed that there was no statistically significant difference between the responses to this insecticide compared with deltamethrin over the entire $8 \mathrm{hr}$ monitoring period (ANOVA: $\mathrm{df}=1 ; \mathrm{F}=3.33 ; \mathrm{P}=0.08$ ), although the rate of knockdown induced by lambdacyhalothrin was significantly higher than that induced by deltamethrin for the period $60 \mathrm{~min}$ to $8 \mathrm{hrs}$ (ANOVA: $\mathrm{df}=1 ; \mathrm{F}=6.55 ; \mathrm{P}=0.02$ ) (Figure 2).

Experiments carried out on bendiocarb showed no difference between either locality compared with each other or with their corresponding susceptible FANG (ANOVA: $\mathrm{df}=1 ; \mathrm{P}>0.05$ in all cases) (Figures 3, 6 and 7).

\section{Synergist bioassays}

These assays [20] were performed on 2-5 day old $F_{1}$ progeny from both Zambia and Zimbabwe using piperonyl butoxide (PBO) as the synergist for P450 mono-oxygenase

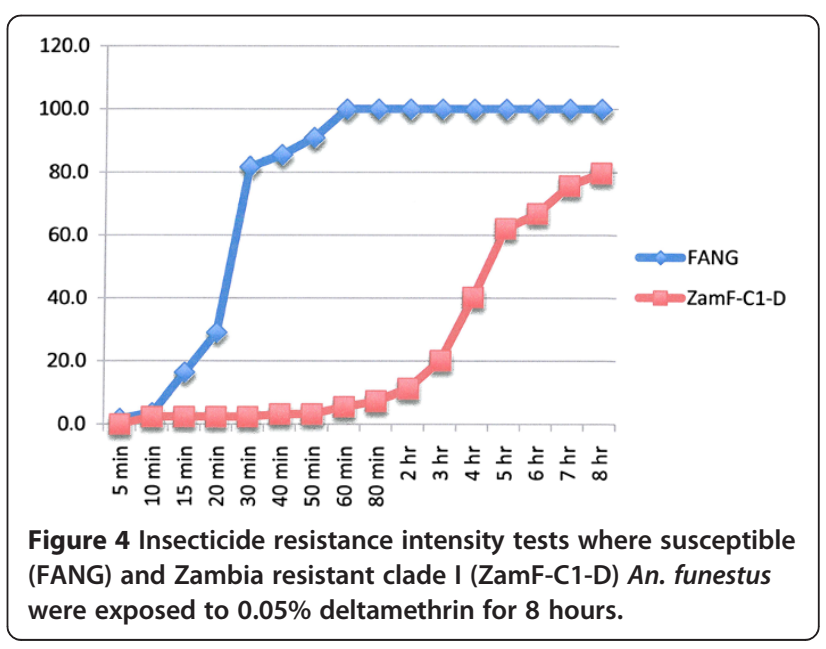




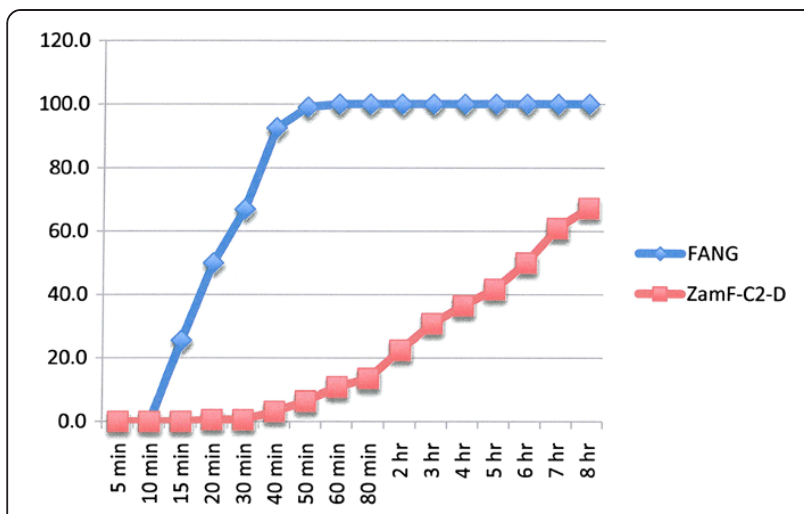

Figure 5 Insecticide resistance intensity tests where susceptible (FANG) and Zambia resistant clade II (ZamF-C2-D) An. funestus were exposed to $0.05 \%$ deltamethrin for 8 hours.

enzymes. Table 5 summarises the results of the synergist bioassays. Pre-exposure to PBO completely nullified deltamethrin resistance in all three samples/clades (Chi-square: $\mathrm{df}=2 ; \mathrm{X}^{2}=7.83 ; \mathrm{P}=0.02$ ), and almost completely nullified bendiocarb resistance in all three samples/clades (Chi-square: $\mathrm{df}=2 ; \mathrm{X}^{2}=43.8 ; \mathrm{P}<0.01$ ).

\section{Discussion and conclusions}

Our data show that An. funestus clade II is not confined to southern Mozambique and Madagascar [16,17] and can be found as far north as Nchelenge District in Zambia, being 2,000 km further north than the current distribution of Chibuto in Mozambique [17]. We do not have data on seasonal abundance of the two clades, so it is possible that clade II also occurs in the Honde Valley, Zimbabwe, but was not detected due to its very low frequency at both times of collection. The frequency of clades I and II in Nchelenge District remained stable over the two years of sampling. Neither the insecticide resistance data nor the sporozoite infection rates provided clear evidence that the

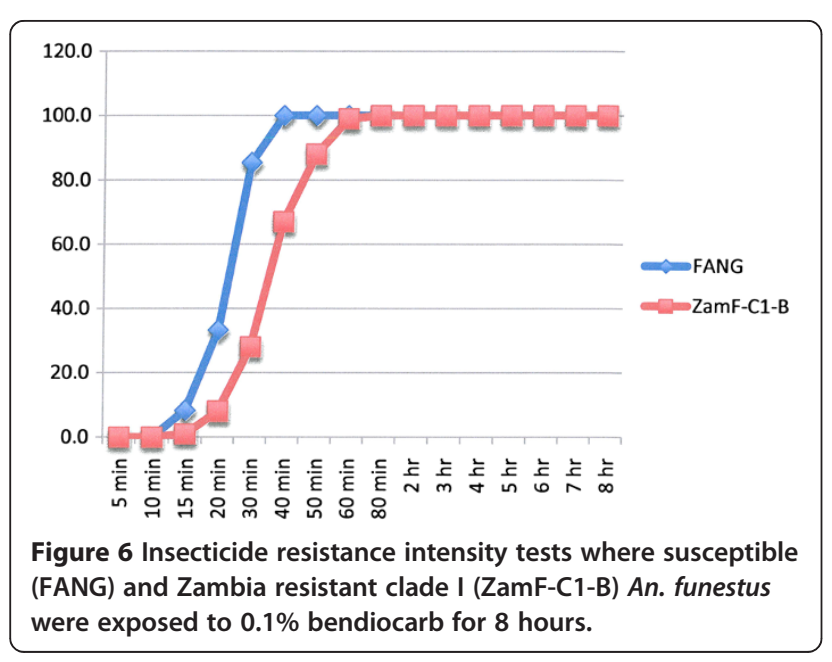

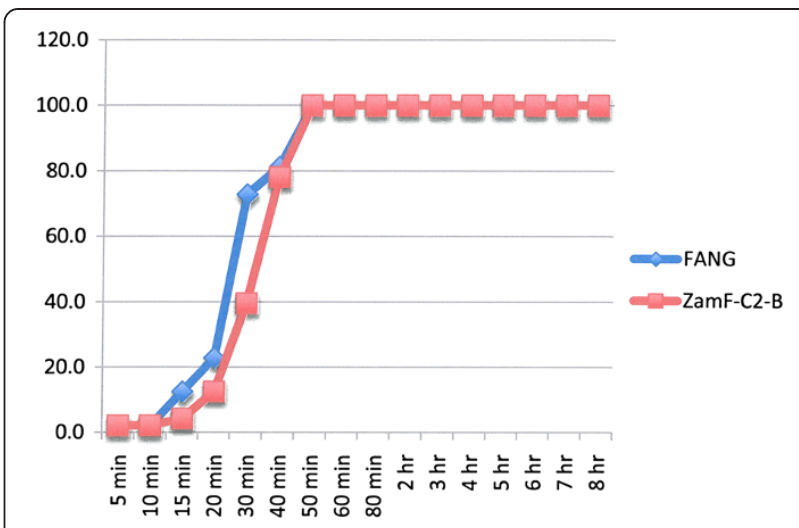

Figure 7 Insecticide resistance intensity tests where susceptible (FANG) and Zambia resistant clade II (ZamF-C2-B) An. funestus were exposed to $0.1 \%$ bendiocarb for 8 hours.

clades are anything other than a polymorphism in a single panmictic population.

The Plasmodium falciparum infection rates for the 2013 Zimbabwe (2.3\%) and Zambia (2.1\%) populations were similar overall, with no significant difference between clade I (8/346 - $2.3 \%)$ and clade II (1/76 - 1.3\%) (Pearson's chi-square $=0.34, \mathrm{P}=0.56$ ). However, there was a 4 -fold increase in sporozoite rates in Nchelenge District in 2014, corresponding with an increase in reported malaria cases (ICEMR data, personal communication). A slight increase was also seen in the 2014 Zimbabwe sample. Of the eight households sampled almost every single inhabitant had had at least one bout of malaria during the 2013-2014 summer. This recent increasing trend of infective (and resistant) An. funestus being associated with increased

Table 4 Resistance intensity assays of Anopheles funestus females by insecticide and strain/clade

\begin{tabular}{llll}
\hline Insecticide & Strain/clade & N (replicates) & $\begin{array}{l}\text { Mean \% knock-down } \\
\text { (SE) after } 8 \text { hrs } \\
\text { exposure }\end{array}$ \\
\hline Deltamethrin & FANG & $179(7)$ & 100 \\
& FUZIM & $192(8)$ & $56.9(3.9)$ \\
& ZAMF C1 & $124(5)$ & $80.9(6.4)$ \\
& ZAMF C2 & $156(6)$ & $67.2(1.9)$ \\
Bendiocarb & FANG & $113(5)$ & 100 \\
& FUZIM & $183(9)$ & 100 \\
& ZAMF C1 & $100(4)$ & 100 \\
& ZAMF C2 & $96(4)$ & 100 \\
Lambda-cyhalothrin & FANG & $18(1)$ & 100 \\
& FUZIM & $106(4)$ & $87.9(3.1)$
\end{tabular}

Mean percentage knock-downs after 8 hour exposures are shown with standard errors (SE) in parentheses where appropriate. FANG = insecticide susceptible laboratory strain; FUZIM = F1 progeny from wild-caught females from Zimbabwe; ZAMF C1 = F1 progeny from wild-caught clade I females from Zambia; ZAMF C2 = F1 progeny from wild-caught clade II females from Zambia; $\mathrm{N}=$ sample size with number of replicates in parentheses. 
Table 5 Synergist experiments on An. funestus clades I and II from Zimbabwe (Honde Valley) and Zambia (Nchelenge)

\begin{tabular}{|c|c|c|c|}
\hline & $\begin{array}{l}\text { Zimbabwe Clade I } \\
\% \text { mortality (n) }\end{array}$ & $\begin{array}{l}\text { Zambia Clade I } \\
\% \text { mortality (n) }\end{array}$ & $\begin{array}{l}\text { Zambia Clade II } \\
\% \text { mortality (n) } \\
\end{array}$ \\
\hline Deltamethrin & $44.0(50)$ & $19.2(26)$ & $29.6(54)$ \\
\hline $\begin{array}{l}\text { Deltamethrin + } \\
\text { PBO }\end{array}$ & $100(97)$ & $100(84)$ & $100(67)$ \\
\hline Bendiocarb & $85.2(57)$ & $6.7(45)$ & $34.0(47)$ \\
\hline $\begin{array}{l}\text { Bendiocarb + } \\
\text { PBO }\end{array}$ & $100(56)$ & $84.1(44)$ & $84.8(46)$ \\
\hline Control PBO & $5.1(79)$ & $3.8(52)$ & - \\
\hline
\end{tabular}

$\%$ mortality = percentage mortality 24 -hr post-exposure. $(n)=$ number of mosquitoes exposed.

number of malaria cases has been reported in other countries, such as Tanzania [24], Kenya [25] and Senegal [26]. These studies underline the importance of An. funestus as a major malaria vector that is sometimes underestimated by researchers and control programmes in Africa.

At present, malaria vector control programmes in Africa rely heavily on chemical methods, with long-lasting insecticide treated bed nets (LLINs) and indoor house spraying with residual insecticides (IRS) being the most widely implemented. However, the alarming increase in insecticide resistance in the main malaria vectors to many of the insecticides used in control, such as pyrethroids, carbamates and DDT, poses a very serious concern $[27,28]$. The susceptibility tests clearly showed that both these An. funestus populations are highly resistant to pyrethroids and carbamates while remaining susceptible to DDT and the organophosphates. The malaria vector control interventions at both localities include distribution of LLINs and in the Honde Valley, the houses had been sprayed with lambda-cyhalothrin in December 2013. Neither intervention had any apparent impact on malaria transmission.

The so-called resistance intensity test carried out here was not a standard method of measuring resistance. While the CDC bottle bioassay method [29] recommends that exposures be continued beyond the diagnostic time in order to assess resistance intensity, they recommend that experiments be terminated at 2 hours and the manual [29] does not give criteria for evaluating resistance intensity. At the 2-hr interval, both An. funestus populations showed mortality of less than $20 \%$ on the WHO diagnostic dose of $0.05 \%$ deltamethrin indicating an extremely high level of resistance. What this means in the field, however, is difficult to judge since these tests do not take into account repellent effects or whether an individual mosquito will indeed rest continuously for 8 hours, or even 1 hour on a treated surface. What is clear however, is that pyrethroid-resistant $A n$. funestus is quite capable of withstanding prolonged exposure to the pyrethroids used for treating bed nets and for house spraying.
The problem of insecticide resistance in An. funestus is no longer a localized one. The resistant population first detected in South Africa [30] and southern Mozambique [20] has now spread to northern Malawi [31] and is affecting the region as a whole, including the localities sampled in this study. The evidence that we have indicates that the resistance mechanisms are mediated by metabolic monooxygenase enzymes, well documented in other An. funestus populations [1]. This metabolic system does not incur a fitness cost [32] and even in the absence of insecticide pressure, the resistance is not lost, facilitating the geographical spread of resistance alleles. However, the resistance in An. funestus populations is by no means uniform across southern Africa with recent studies in southeastern Zambia showing resistance to DDT as well as pyrethroids and carbamates [33].

This places yet more constraints on the vector control programmes operating in the region with only the organophosphates having a generalised killing effect on the $A n$. funestus populations. While both Zambia and Zimbabwe are changing their IRS policies and will be using an organophosphate for IRS in the coming transmission season, the need for additional, affordable, new tools for vector control [28] has acquired an urgency as never before.

\section{Competing interests}

The authors declare that they have no competing interests.

\section{Authors' contributions}

$\mathrm{RH}, \mathrm{MC}, \mathrm{LN}, \mathrm{OW}, \mathrm{MM}$ and $\mathrm{AM}$ carried out the field data collection, insecticide resistance and synergist assays. KC, RC, LN and EA carried out the molecular experiments and analyzed the data. SM and MM facilitated the fieldwork in Zimbabwe and Zambia respectively. $\mathrm{RH}, \mathrm{LK}, \mathrm{BB}$ and $\mathrm{MC}$ contributed to the study design and data analysis. All authors contributed to drafting the manuscript and approved the final version.

\section{Acknowledgments}

We are grateful to the District Health Team of Mutasa District, the Provincial Health Team of Manicaland Province, the National Malaria Control

Programme and the Ministry of Health and Child Welfare, for facilitating the field work in Zimbabwe, and to the District Community Health Office of Nchelenge, the Provincial Health Office of Luapula Province, the National Malaria Control Programme and the Ministry of Health for facilitating data collection in Zambia. This work was supported by the Division of

Microbiology and Infectious Diseases, National Institutes of Allergy and Infectious Diseases, National Institutes of Health, as part of the International Centers of Excellence for Malaria Research (U19 Al089680). MC is supported by the South African DST/NRF Research Chair initiative.

This work was undertaken for the Southern Africa International Centers of Excellence for Malaria Research, which includes the following institutions: Johns Hopkins Malaria Research Institute, Baltimore, USA; Tropical Diseases Research Centre, Ndola, Zambia; Macha Research Trust, Macha, Zambia; University of Zambia, Lusaka, Zambia; the Biomedical Research and Training Institute, Harare, Zimbabwe; the National Institute of Health Research, Harare, Zimbabwe; the Wits Research Institute for Malaria, Johannesburg, South Africa.

\section{Author details}

${ }^{1}$ Wits Research Institute for Malaria, School of Pathology, Faculty of Health Sciences, University of the Witwatersrand, Johannesburg, South Africa. ${ }^{2}$ Centre for Opportunistic, Tropical and Hospital Infections, National Institute for Communicable Diseases, National Health Laboratory Service, 
Johannesburg, South Africa. ${ }^{3}$ School of Life Sciences, College of Natural Sciences, Kyungpook National University, Daegu 702-701, Korea. ${ }^{4}$ Tropical Diseases Research Centre, Ndola, Zambia. ${ }^{5}$ Biomedical Research and Training Institute, Harare, Zimbabwe. ${ }^{6}$ National Institute for Health Research, Harare, Zimbabwe.

Received: 9 July 2014 Accepted: 27 September 2014

Published online: 08 October 2014

\section{References}

1. Coetzee M, Koekemoer LL: Molecular systematics and insecticide resistance in the major African malaria vector Anopheles funestus. Annu Rev Entomol 2013, 58:393-412.

2. Coetzee M, Hunt RH, Wilkerson R, Della Torre A, Coulibaly MB, Besansky NJ: Anopheles coluzzii and Anopheles amharicus, new members of the Anopheles gambiae complex. Zootaxa 2013, 3619:246-274.

3. Sinka ME, Bangs MJ, Manguin S, Coetzee M, Mbogo CM, Hemingway J, Patil AP, Temperley WH, Gething PW, Kabaria CW, Okara RM, Van Boeckel T, Godfray HCJ, Harbach RE, Hay SI: The dominant Anopheles vectors of human malaria in Africa, Europe and the Middle East: occurrence data, distribution maps and bionomic précis. Parasit Vectors 2010, 3:117.

4. Brown AWA: Insecticide resistance in mosquitoes: a pragmatic review. J Am Mosa Control Assoc 1986, 2:123-140.

5. Coetzee M, Kruger P, Hunt RH, Durrheim DN, Urbach J, Hansford CF: Malaria in South Africa: 110 years of learning to control the disease. Sth Afr Med J 2013, 103(10 Suppl 2):770-778.

6. Gillies MT, De Meillon B: Anophelinae of Africa South of the Sahara. Johannesburg, South Africa: Publ Sth Afr Inst Med Res; 1968:54.

7. Gillies MT, Coetzee M: A Supplement to the Anophelinae of Africa South of the Sahara (Afrotropical Region). Johannesburg, South Africa: Publ Sth Afr Inst Med Res; 1987:55.

8. Wilkes TJ, Matola YG, Charlwood JD: Anopheles rivulorum, a vector of human malaria in Africa. Med Vet Entomol 1996, 10:108-110.

9. Kawada H, Dida GO, Sonye G, Njenga SM, Mwandawiro C, Minakawa N: Reconsideration of Anopheles rivulorum as a vector of Plasmodium falciparum in Western Kenya: some evidence from biting time, blood preference, sporozoite positive rate, and pyrethroid resistance. Parasit Vectors 2012, 5:230.

10. Green $\mathrm{CA}$, Hunt $\mathrm{RH}$ : Interpretation of variation in ovarian polytene chromosomes of Anopheles funestus Giles, A. parensis Gillies, and A. aruni?. Genetica 1980, 51:187-195.

11. Green CA: Cladistic analysis of chromosome data (Anopheles (Cellia) Myzomyia). J Hered 1982, 73:2-11.

12. Spillings BL, Brooke BD, Koekemoer LL, Chiphwanya J, Coetzee M, Hunt RH: A new species concealed by Anopheles funestus Giles, the major malaria vector in Africa. Am J Trop Med Hyg 2009, 81:510-515.

13. Costantini C, Sagnon NF, Ilboudo-Sanogo E, Coluzzi M, Boccolini D: Chromosomal and bionomic heterogeneities suggest incipient speciation in Anopheles funestus from Burkina Faso. Parassitologia 1999, 41:595-611.

14. Michel AP, Guelbeogo WM, Grushko O, Schemerhorn BJ, Kern M, Willard MB, Sagnon NF, Costantini C, Besansky NJ: Molecular differentiation between chromosomally defined incipient species of Anopheles funestus. Insect Mol Biol 2005, 14:375-387.

15. Koekemoer LL, Kamau L, Hunt RH, Coetzee M: A cocktail polymerase chain reaction assay to identify members of the Anopheles funestus (Diptera: Culicidae) group. Am J Trop Med Hyg 2002, 66:804-811.

16. Michel AP, Ingrasci MJ, Schemerhorn BJ, Kern M, Le Goff G, Coetzee M, Elissa N, Fontenille D, Vulule J, Lehmann T, Sagnon NF, Costantini C, Besansky NJ: Rangewide population genetic structure of the African malaria vector Anopheles funestus. Mol Ecol 2005, 14:4235-4248.

17. Choi KS, Koekemoer LL, Coetzee M: Population genetic structure of the major malaria vector Anopheles funestus s.s. and allied species in southern Africa. Parasit Vectors 2012, 5:283.

18. World Health Organization: Test procedures for insecticide resistance monitoring in malaria vector mosquitoes. Geneva: WHO; 2013.

19. Hunt RH, Coetzee M: A parasitological, cytogenetic and biochemical study of Anopheles gambiae (Diptera: Culicidae) from the Peoples Republic of Congo. Sth Afr Med J 1989, 76:362-364.

20. Brooke BD, Kloke G, Hunt RH, Koekemoer LL, Temu EA, Taylor ME, Small G, Hemingway J, Coetzee M: Bioassay and biochemical analyses of insecticide resistance in southern African Anopheles funestus (Diptera: Culicidae). Bull Entomol Res 2001, 91:265-272.

21. Choi KS, Coetzee M, Koekemoer LL: Detection of clade types (clades I and II) within Anopheles funestus sensu stricto by the hydrolysis probe analysis (Taqman assay). Parasit Vectors 2013, 6:173.

22. Burkot TR, Williams JL, Schneider I: Identification of Plasmodium falciparum-infected mosquitoes by a double antibody enzyme-linked immunosorbent assay. Am J Trop Med Hyg 1984, 33:783-788.

23. Durnez L, Van Bortel W, Denis L, Roelants P, Veracx A, Trung HD, Sochantha T, Coosemans M: False positive circumsporozoite protein ELISA: a challenge for the estimation of the entomological inoculation rate of malaria and for vector incrimination. Malar J 2011, 10:195.

24. Lwetoijera DW, Harris C, Kiware SS, Dongus S, Devine GJ, McCall PJ, Majambere S: Increasing role of Anopheles funestus and Anopheles arabiensis in malaria transmission in the Kilombero Valley. Tanzania Malar J 2014, 13:331

25. McCann RS, Ochomo E, Bayoh MN, Vulule JM, Hamel MJ, Gimnig JE, Hawley WA, Walker ED: Reemergence of Anopheles funestus as a vector of Plasmodium falciparum in Western Kenya after long-term implementation of insecticide treated bed nets. Am J Trop Med Hyg 2014, 90:597-604.

26. Trape J-F, Tall A, Diagne N, Ndiath O, Ly AB, Faye J, Dieye-Ba F, Roucher C, Bouganali C, Badiane A, Sarr FD, Mazenot C, Toure-Balde A, Raoult D, Druilhe P, Mercereau-Puijalon O, Rogier C, Sokhna C: Malaria morbidity and pyrethroid resistance after the introduction of insecticide-treated bednets and artemisinin-based combination therapies: a longitudinal study. Lancet Infect Dis 2011, 11:925-932.

27. Rivero A, Vezilier J, Weill M, Read AF, Gandon S: Insecticide control of vector-borne diseases: when is insecticide resistance a problem? PLoS Pathog 2010, 6(8):e1001000.

28. Hemingway J: The role of vector control in stopping the transmission of malaria: threats and opportunities. Phil Trans R Soc B 2014, 369:20130431.

29. CDC: Guideline for evaluating insecticide resistance in vectors using the CDC bottle bioassay. Atlanta: Centers for Disease Control \& Prevention, US Dept Health \& Human Services; 2010.

30. Hargreaves K, Koekemoer LL, Brooke BD, Hunt RH, Mthembu J, Coetzee M: Anopheles funestus is resistant to pyrethroid insecticides in South Africa. Med Vet Entomol 2000, 14:181-189.

31. Hunt RH, Edwardes M, Coetzee M: Pyrethroid resistance in southern African Anopheles funestus extends to Malawi. Parasit Vectors 2010, 3:122

32. Okoye PN, Brooke BD, Hunt RH, Coetzee M: Relative developmental and reproductive fitness associated with pyrethroid resistance in the major southern African malaria vector Anopheles funestus. Bull Entomol Res 2007, 97:599-605.

33. Chanda E, Hemingway J, Kleinschmidt I, Rehman AM, Ramdeen V, Phiri FN, Coetzer S, Mthembu D, Shinondo CJ, Chizema-Kawesha E, Kamuliwo M, Mukonka V, Baboo KS, Coleman M: Insecticide resistance and the future of malaria control in Zambia. PLoS One 2011, 6(9):e24336.

doi:10.1186/s13071-014-0464-z

Cite this article as: Choi et al:: Insecticide resistance and role in malaria transmission of Anopheles funestus populations from Zambia and Zimbabwe. Parasites \& Vectors 2014 7:464

\section{Submit your next manuscript to BioMed Central and take full advantage of:}

- Convenient online submission

- Thorough peer review

- No space constraints or color figure charges

- Immediate publication on acceptance

- Inclusion in PubMed, CAS, Scopus and Google Scholar

- Research which is freely available for redistribution 